\title{
Corona Virus and the General Adaptation Syndrome
}

Derrick Lonsdale, MBBS, FAAP, FACN ${ }^{*}$

Associate Emeritus, Cleveland Clinic, Cleveland, Ohio, USA

\section{Introduction}

Louis Pasteur played a major part in the discovery of microorganisms, particularly those that affect health. He is purported to have said on his deathbed "I was wrong: It is the terrain that matters". He meant, of course, the natural defenses with which the human body is equipped. All members of the animal kingdom live in a hostile environment, the major contributors being the microorganisms that result in disease. They can be thought of as "the enemy" representing a war-like attack that tests our naturally endowed defensive mechanisms. The paradigm in medicine that exists at present is "kill the enemy". This attack is automatically met by a complex of defenses that can be viewed as much like a war. The question of what can be done to make sure that the defenses are as vigorous as possible is answered by the work of Hans Selye.

\section{Stress}

As a medical student in Hungary, Selye was exposed to a group of patients suffering from the various diseases with which they had been diagnosed. Instead of listening to the teacher, he observed the facial expression of each of the patients as they were presented. He came to the conclusion that they all looked very much the same, that it was a response to the stress imposed by the illness from which they were suffering. After graduation, he emigrated to Canada and set up an Institute in Montréal with the specific intention of studying the effects of stress, defined as anything that attacked the status quo of an animal. He stressed thousands of rats with various forms of trauma and infections. He concluded that if the animal was fit, it would adapt to or resist whatever stress was imposed. If it failed to adapt, or if the stress was overwhelming, the animal would die. He called it the General Adaptation Syndrome (GAS) [1]. He found that the various laboratory studies on the blood and tissues of the injured animals exactly replicated the information obtained from laboratory studies done on sick humans and called human diseases "The diseases of adaptation". One of his remarkable conclusions was that this adaptation through the GAS required huge amounts of energy. In Selye's time, little was known about energy metabolism. However, one of his students knew that thiamine was vital for energy generation and he was able to show that deficiency of this vitamin resulted in a replication of the GAS, without traumatizing the animal [2]. We can conclude that a severe lack of thiamine might be the cause of what we call "shock" and a complete lack would be lethal. Today we have detailed knowledge concerning the role played by thiamine in the generation of cellular energy and this particularly applies to the part of the brain that organizes and controls our adaptive ability through the autonomic and endocrine systems.

\section{Does Selye's Work Apply to the Corona virus?}

A recent discovery is that a combination of hydrocortisone, ascorbic acid and thiamine (HAT therapy) given intravenously, is a successful treatment for sepsis, a condition that is almost uniformly lethal [3]. By damping down the associated inflammation and stimulating oxidative metabolism in the production of energy, this is clearly a defensive treatment. Recently thiamine has also been found to be essential in the treatment of people with critical disease [4], strongly suggesting that energy is necessary for a successful outcome. It has been reported that in-patients being treated for psychiatric symptoms, are at risk for developing the serious symptoms of Wernicke Encephalopathy, well known to be due to thiamine deficiency [5]. A report from the Department of Infectious Diseases, Wenzhous Central Hospital, Zhejiang Province, China describes the symptoms of the patients with Coronavirus treated in that hospital. Nausea and vomiting were described in some of the patients [6]. Although never pathognomonic, they occur in beriberi, the best known thiamine deficiency disease. One of the common findings in the Corona virus patients was hypokalemia, caused by defective action of the sodium/potassium cellular membrane pump [7]. Hypokalemia occurs in beriberi, perhaps the best known condition primarily associated with energy failure.

\section{The Significance}

It is proposed here that stimulating energy metabolism might improve the defensive action organized and conducted

*Corresponding author: Derrick Lonsdale, MBBS, FAAP, FACN, Associate Emeritus, Cleveland Clinic, Cleveland, Ohio, 28575 Westlake Village, Dr. Westlake $\mathrm{OH} 44145$, USA, Tel: 440-4714852

Received: April 18, 2020

Accepted: June 04, 2020

Published online: June 06, 2020

Citation: Lonsdale D (2020) Corona Virus and the General Adaptation Syndrome. Sch J Emerg Med Crit Care 4(1):73-74 
by the brain, obeying the dictum suggested by Louis Pasteur. It assumes that the genetics of the patient decide the intricacies of the defense program, but the relatively new science of epigenetics shows that energy, derived from nutrition, can improve genetic status. We believe that we have shown evidence that thiamine and magnesium supplementation are inherently necessary in a population in which nutrition is imperfect [8].

\section{Thiamine Tetrahydrofurfuryl Disulfide (TTFD)}

TTFD is the synthetic equivalent of allithiamine, a naturally occurring disulfide derivative of thiamine [9]. Well studied by Japanese researchers, it partially protected TTFD pre-treated mice from cyanide poisoning [10]. The total body stores of thiamine are relatively small and deficiency can develop in patients undergoing acute metabolic stress [11].

It is suggested that a clinical trial with this agent might help to improve all the defensive strategies required to meet the pathologic consequences of the Covid virus, or any other infection. There is considerable evidence that high calorie malnutrition is common in America and it would be expected that this would weaken immune responses [8]. The vagus nerve has an important role in regulation of metabolic homeostasis and immune function [12], is dependent on the acetylation of choline from the function of the citric acid cycle which is in turn dependent on the entry of glucose catalyzed by thiamine.

\section{References}

1. Selye $H$ (1946) The general adaptation syndrome and the diseases of adaptation. J Clin Endocrinol Metabol 6: 117-230.
2. Skelton FR (1950) Some specific and some non-specific effects of thiamine deficiency in the rat. Proc Soc Exp Biol Med 73: 516519.

3. Marik PE (2018) Hydrocortisone, ascorbic acid and thiamine (HAT therapy) for the treatment of sepsis. Nutrients 10: 1762.

4. Polegato BF, Pereira AG, Azevedo PS, et al. (2019) Role of thiamin in health and disease. Nutr Clin Pract 34: 558-564.

5. Guirguis M, Manning S, Chave M, et al. (2017) Are psychiatric inpatients, at risk of developing WE, being identified and adequately treated? Primary Care Companion CNS Discord 19.

6. Chen D, Li X, Song Q, et al. (2019) Hypokalemia and clinical implications in patients with Coronavirus Disease 2019 (COV ID 19). Department of Infectious Diseases, Wenzhou Central Hospital, Zhejiang Province, China.

7. Pirahanchi Y, Aeddula NR (2020) Physiology, sodium potassium. In: Stat Pearls [Internet]. Stat Pearls publishing, Treasure Island $\mathrm{Fl}$.

8. Lonsdale D, Marrs C (2017) Thiamine deficiency disease, dysautonomia and high calorie malnutrition. Academic Press, London.

9. Lonsdale D (2004) Thiamine tetrahydrofurfduryl disulfide: A little known therapeutic agent. Med Sci Monit 19: 199-203.

10. Shimazono N, Katsura E (1965) Beriberi and Thiamine. Igvaku Shoin Ltd, Tokyo.

11. Attaluri P, Castillo A, Edriss H, et al. (2018) Thiamine deficiency: an important consideration in critically ill patients. Am J Med Sci 356: 382-390.

12. Pavlov VA, Tracey KJ (2012) The vagus nerve and the inflammatory reflex-linking immunity and metabolism. Nat Rev Endocrinol 8: 743-754.

DOI: $10.36959 / 592 / 385$ 